\title{
Barotropic Instability of Midlatitude Zonal Jets on Mars, Earth and Venus
}

\author{
DiANe V. Michelangeli ANd RichaRd W. ZUREK* \\ Department of Geological and Planetary Sciences, California Institute of Technology, Pasadena, CA 91125
}

LEE S. ELSON

Jet Propulsion Laboratory, California Institute of Technology, Pasadena, CA 91109

(Manuscript received 13 June 1986, in final form 2 February 1987)

\section{ABSTRACT}

\begin{abstract}
We have used a linearized nondivergent barotropic vorticity model on a sphere to intercompare the fastest growing, barotropically unstable wave modes computed for zonal jets at high latitudes in the middle atmospheres of Venus, Earth, and Mars. Such zonal jets have been observed in the wintertime stratosphere on Earth and have been inferred from remotely sensed temperatures in the Venus middle atmosphere and in the wintertime Martian atmosphere. The comparison was done by extending the results of Hartmann for his simple analytic profile of a latitudinally varying terrestrial zonal wind to zonal wind profiles characterized by the larger Rossby numbers (Ro) appropriate to Mars and Venus. As Hartmann's results suggested, the fastest growing barotropic waves continue to grow more quickly as Ro increases. Eventually, the fastest growing mode shifts from a zonal wavenumber $k=1$ to a $k=2$ mode, both located on the poleward flank of the high-latitude jet. However, for somewhat higher Rossby numbers, the $k=2$ mode on the equatorward side of the zonal jet becomes the fastest growing planetary-scale barotropic mode, and this transition is marked by a discontinuous shift to longer wave periods. The Venus high-latitude zonal jet appears remarkably close to this transition Ro. For each of the three planets, satellite-borne instruments have detected wave patterns in the thermal radiance field in the vicinity of the high-latitude zonal jets. As reported earlier for the terrestrial wintertime stratosphere by Hartmann and for Venus by Elson, these observed waves have characteristics similar to those computed for the fastest growing barotropic modes. For Mars, we find that such modes would have zonal wavenumbers 1 or 2 , with $e$-folding times of 2-3 days and periods of $0.75-2.5$ days; the longer period $(k=2)$ equatorward mode would dominate for the faster and narrower zonal jets. A poleward mode with $k=1$ and a period of 1.2 days is the barotropic mode most likely to be consistent with the Mariner-9 IRIS observations of thermal waves above the I mb $(\sim 20$ $\mathrm{km})$ level in the Martian atmosphere.
\end{abstract}

\section{Introduction}

Recent analyses of satellite radiance data have indicated the presence of planetary-scale waves in the middle atmospheres of Earth (Venne and Stanford, 1979, 1982), Venus (Taylor et al., 1980), and Mars (Conrath, 1981; Martin and Kieffer, 1979). Often these large-scale waves exist where the latitudinal shear of the zonal wind is large, and this suggests that barotropic instability may be an important process in these regions.

For Venus, Elson (1982) used the linearized barotropic nondivergent vorticity equation to find the fastest growing, barotropically unstable wave mode. This mode had zonal wavenumber 2 and a period of about three terrestrial days, both characteristic of the highlatitude thermal wave observed in the upper-cloud region of Venus by the Pioneer Venus Orbiter Infrared Radiometer (OIR). In a similar manner, Hartmann

* Permanent affiliation: Jet Propulsion Laboratory, California Institute of Technology, Pasadena, CA 91109.
(1983) found that barotropic instability in the terrestrial stratosphere could produce a wave of the "4-day" variety detected by Venne and Stanford (1979). Using a quasi-geostrophic model on a sphere, Hartmann (1983) also investigated the effects of divergence and of vertical shear of the basic state zonal wind on the barotropically unstable modes. The period, relative $e$-folding time and zonal wavenumber of the most unstable mode were relatively unchanged by the inclusion of the velocity divergence and vertical shear in the model. The most important effects of the vertical variation of the zonal jet were that the absolute $e$-folding time of the fastest growing, wavenumber 1 wave increased by nearly $50 \%$, and polar modes having larger longitudinal wavenumbers were no longer found to be unstable. Pfister (1979) had previously found, using a $\beta$-plane model, that the growth rates computed for essentially barotropic modes were markedly reduced when realistic vertical shear was included. For Mars, Conrath (1981) argued that the wave-like perturbations found in the Mariner-9 temperature data for high latitudes during winter were more likely to be produced by vertical propagation of 
nearly stationary waves rather than by in situ instabilities, since the phase speeds of locally produced disturbances would be much faster than indicated by the observations. However, Conrath also noted that the thermal wind profile was probably barotropically unstable and that the observational coverage provided by Mariner- 9 was subject to aliasing. Thus, barotropic instability was still a candidate mechanism.

In the work reported here, a linearized barotropic nondivergent vorticity model is used to find the fastest growing, barotropically unstable mode for zonal wind profiles which may be representative of the Martian high latitudes during winter. Our numerical method finds only the fastest growing mode for a given zonal wavenumber. These modes are the most likely to be observed, and Hartmann's (1983) analyses indicated that their computed period and spatial variations pro-vide a reasonable first approximation to the streamfunction fields of their quasi-divergent counterparts. This is critical to our use of the nondivergent vorticity equation, since it is the quasi-divergent modes which can give rise to the patterns of thermal emission observed remotely by satellites. The same analytic form for the high-latitude jet is used here as in Hartmann. This facilitates comparison with his work, while also providing a reasonably good fit to the thermal winds constructed for Mars from the Mariner-9 Infrared Interferometer Spectrometer (IRIS) temperature profiles and for Venus from the Pioneer Venus OIR data.

The high-latitude zonal jet on Venus and the polarnight jets in the atmospheres of Earth and Mars all appear to be of comparable angular width and position. If the effective width and location of the jet are fixed, the linearized, nondivergent vorticity equation has one free parameter, the Rossby parameter Ro $\equiv U / \Omega a$, with $U$ a characteristic velocity of the zonal jet, the planetary radius $a$, and the sidereal frequency $\Omega$ of the planet's solid body rotation or, in the case of Venus, of the solid body component of its atmospheric motion. Because Mars is a smaller planet and both the atmosphere and the surface of Venus rotate relatively slowly, the highlatitude zonal jets on Mars and Venus are characterized by larger Ro than on Earth. Thus, our results for Mars are complementary to Hartmann's (1983) parametric studies for Earth's stratosphere and can'be presented in the context of that larger parameter space. Hartmann's results showed that the growth rate of a barotropically unstable mode with zonal wavenumber $k$ $=2$ became comparable to the fastest growing $k=1$ mode for the strongest jets. Our calculations show that the $k=2$ mode does not become the fastest growing barotropic wave until the Ro of these relatively broad jets is increased to values characteristic of Mars (Ro $\sim 0.4$ ). Both the $k=1$ and $k=2$ modes have their maximum amplitude poleward of the zonal wind maximum for this Ro range. However, at the somewhat larger Ro which may be characteristic of the strongest jets on Mars or Venus, the fastest growing barotropic mode is a $k=2$ mode whose maximum amplitude is now on the equatorward flank of the jet. Hartmann had previously found midlatitude modes associated with the region of negative absolute vorticity on the equatorward flank of the high-latitude zonal jets, but these were never the fastest growing barotropic modes for the terrestrial stratosphere. This general variation with increasing Ro for fixed position and width of the jet is discussed in section 4 .

First, however, we briefly describe in section 2 the barotropic nondivergent vorticity model used to characterize the fastest growing modes. Then, in section 3 , we select a range of zonal jet parameters-primarily Ro and the jet width-appropriate to Venus and to Mars. Section 4 follows with a unified presentation (in terms of the Ro parameter) of our results with those of Elson (1982) and Hartmann (1983) for nondivergent, barotropically unstable modes of relatively broad, midlatitude zonal jets in planetary atmospheres. Section 5 presents some specific examples appropriate to Venus and Mars.

\section{Barotropic model}

Following Hartmann (1983), we represent the zonalmean zonal wind $\bar{u}$ by this simple analytic form:

$$
\bar{u}=U_{0} \eta(\theta) \cos \theta, \quad \eta \equiv \operatorname{sech}\left[\frac{2}{B}\left(\theta-\theta_{0}\right)\right]
$$

with latitude $\theta$ and constant jet parameters $U_{0}, \theta_{0}, B$. Given the planetary radius $a, U_{0} a^{-1}$ is the maximum angular velocity of the zonal jet, $\theta_{0}$ is the latitude of that maximum, and $B$ is the width of the jet. The zonal velocity maximum is also near $\theta_{0}$ and its value is roughly $U_{0} \cos \theta_{0}$. For time $t$ and east longitude $\lambda$, the perturbation streamfunction $\psi^{\prime}$ is assumed to have the form:

$$
\psi^{\prime}=\psi(\theta) \exp [i k(\lambda-\sigma t)]
$$

with zonal wavenumber $k$ and $\sigma=\sigma_{r}+i \sigma_{i}$ the complex frequency of the disturbance. Given (2) and the analytic form (1), the linearized barotropic nondivergent vorticity equation can be written in the following nondimensional form:

$$
\left(\operatorname{Ro} \eta-\frac{\hat{\sigma}}{2}\right)\left(\frac{d^{2}}{d \theta^{2}}-\tan \theta \frac{d}{d \theta}-\frac{k^{2}}{\cos ^{2} \theta}\right)[\psi]+\frac{\alpha \psi}{\cos \theta}=0
$$

with the nondimensional absolute vorticity gradient

$$
\alpha=\left[1-\operatorname{Ro}\left(\frac{d^{2} \eta}{d \theta^{2}}-3 \tan \theta \frac{d \eta}{d \theta}-2 \eta\right)\right] \cos \theta .
$$

Solutions to (3) for fixed $\theta_{0}$ and $B$ depend only upon the nondimensional Rossby number Ro $\equiv\left(U_{0} / 2 \Omega a\right)$, as long as the period and $e$-folding times are expressed in terms of $\Delta$, the length of the sidereal day; i.e., $\hat{\sigma}$ is $\sigma$ normalized by the planetary rotation rate $\Omega \equiv 2 \pi /$ $\Delta$. (This choice of Ro implies a velocity scale $U=U_{0} / 2$ 
$\sim U_{0} \cos \theta_{0}$.) For Earth and Mars, the $\Omega$ are nearly identical. For Venus $\Omega$ is taken to be $1 / 4$ that of Mars; this assumes that the solid body component of the atmospheric rotation is given by the Venusian "4-day" wind. Specific planetary parameters will lead to different solutions of (1) only if they combine to yield different Rossby parameters. Table 1 lists the different values for Venus, Earth, and Mars of the zonal wind velocity $U_{0}$ which yield the same Rossby parameter, given the planetary parameters in Table 2 .

For this simple model, the zonal-mean zonal wind equation is given by

$$
\frac{\partial \bar{u}}{\partial t}=-\frac{1}{a \cos ^{2} \theta} \frac{\partial}{\partial \theta}\left(\overline{u^{\prime} v^{\prime}} \cos ^{2} \theta\right) \equiv A_{u}
$$

where the overbar denotes a zonal (i.e., longitudinal) average and $u^{\prime}, v^{\prime}$ are the longitudinal perturbation eastward and northward wind components. $A_{u}$, the zonal-mean zonal acceleration induced by the barotropic perturbation, is related to the Eliassen-Palm (EP) flux-divergence $\nabla \cdot F$ by $(a \cos \theta) A_{u}=\nabla \cdot F$, which is also the convergence of the poleward flux of angular momentum. The enstrophy budget consistent with (3) also involves $A_{u}$ (Hartmann, 1983):

$$
\frac{\partial}{\partial t}\left(\frac{1}{2} \overline{\zeta^{\prime} \zeta^{\prime}}\right)=-A_{u}(2 \Omega \alpha / a)
$$

with the perturbation relative vorticity $\zeta^{\prime}=\nabla^{2} \psi^{\prime}$. Thus, the barotropic perturbations will grow if the acceleration $A_{u}$ (or equivalently, the EP flux-divergence) has the opposite sign as the meridional gradient of zonalmean absolute vorticity. As in Hartmann, the unstable perturbations found here are growing at essentially all latitudes. A necessary and often sufficient condition for barotropic instability to occur is that the absolute vorticity gradient $\alpha$ vanish somewhere in the latitudinal domain of interest (Kuo, 1949). For large values of the Rossby parameter Ro (and thus $U_{0}$ for a given planet), the absolute vorticity gradient derived from (1) may vanish on the equatorward side of the zonal jet, as well as on the poleward flank.

The barotropically unstable modes discussed in this paper were computed using the numerical model of Elson (1978). This model integrates the linearized nondivergent barotropic vorticity equation (3) forward in time for a given value of the zonal wavenumber $k$

TABLE 1. The jet parameter $U_{0}\left(\mathrm{~m} \mathrm{~s}^{-1}\right)$ for Mars, Earth and Venus corresponding to various $\mathrm{Ro}=U_{0} / 2 \Omega a$ values.

\begin{tabular}{lrrrrrrrr}
\hline \hline & \multicolumn{8}{c}{ Ro } \\
\cline { 2 - 9 } & 0.10 & 0.13 & 0.20 & 0.26 & 0.41 & 0.5 & 0.58 & 0.62 \\
\hline Mars & 48 & 64 & 96 & 124 & 200 & 240 & 280 & 300 \\
Earth & 93 & 124 & 186 & 240 & 387 & 464 & 541 & 580 \\
Venus & 21 & 28 & 43 & 56 & 89 & 107 & 125 & 134 \\
\hline
\end{tabular}

TABLE 2. Planetary parameters.

\begin{tabular}{lccc}
\hline \hline Parameter & Venus & Earth & Mars \\
\hline$a(\mathrm{~km})$ & 6052 & 6378 & 3394 \\
$\Omega\left(\mathrm{s}^{-1}\right)$ & $1.772 \times 10^{-5 *}$ & $7.292 \times 10^{-5}$ & $7.088 \times 10^{-5}$ \\
$a \Omega\left(\mathrm{m} \mathrm{s}^{-1}\right)$ & 107 & 465 & 241 \\
\hline
\end{tabular}

* Venus rotation frequency is assumed to be that of the atmospheric solid body component near the cloud tops $(\sim 65 \mathrm{~km})$; i.e., the "4day" wind.

and for the absolute vorticity gradient (4) and with $\eta$ given by (1). Eventually, the time-integrated streamfunction field is dominated by the fastest growing mode. The time integration is stopped when the time variation at all latitude points yields essentially the same complex frequency. Here, the perturbations are assumed to be symmetric about the equator and 91 latitudinal gridpoints were used to span the Northern Hemisphere. In this way, the fastest growing, barotropically unstable mode was found, given the zonal wavenumber $k$ and the zonal jet parameters Ro, $\theta_{0}$, and $B$.

\section{Zonal jets: Choice of parameters \\ a. Venus}

Here the zonal-mean zonal wind in the middle atmosphere of Venus is modeled as just the superposition of a midlatitude zonal jet upon a solid-body component of atmospheric rotation. The planetary rotation rate $\Omega_{V}=2 \pi \Delta_{V}{ }^{-1}$ is assumed to be given by the period $\Delta_{V}$ of the solid body component, which is observed to vary significantly with height; it decreases, reaching a minimum of 4 days or so at $65 \mathrm{~km}$, near the cloud tops. Winds above this level are inferred from the observed temperature field by assuming cyclostrophic balance and so are somewhat uncertain. It does appear, however, that $\Delta_{V}$ increases by roughly a factor of 2 by 75 $\mathrm{km}$, and that there is a high-latitude jet which peaks near $\theta=60^{\circ}$ and in the $65-70 \mathrm{~km}$ region, where the amplitude (i.e., $U_{0} \cos \theta_{0}$ ) of the zonal jet relative to the solid body component may range from 50 to $110 \mathrm{~m}$ $\mathrm{s}^{-1}$ (Elson, 1982; Newman et al., 1984; Taylor et al., 1985). These combinations of $\Delta_{V}$ and $U_{0}$ correspond to Ro $\geqslant 0.5$, with Ro $\sim 0.5$ characteristic of the height where planetary-scale waves are observed at high latitudes (Taylor et al., 1980; Elson, 1982). The angular width of the zonal jet is approximately $20^{\circ}$.

\section{b. Mars}

Conrath (1981) analyzed the atmospheric temperature profiles retrieved from the Mariner-9 IRIS measurements of the spectral radiance within the $\mathrm{CO}_{2} 15$ $\mu \mathrm{m}$ absorption band. The observations were taken during January and early February 1972, a period of approximately 40 days spanning late northern winter on Mars. (Note that $L_{s}=330^{\circ}-350^{\circ}$, where $L_{s}$ is the 
areocentric longitude of the sun measured from its position at the beginning of northern spring.) Temperatures were retrieved at five pressure levels $(0.117,0.300$, $0.518,1.13$, and $2.46 \mathrm{mb}$ ) and were grouped into $10^{\circ}$ latitude intervals. When averaged over this entire period, the Mariner- 9 data yield the late northern winter meridional plane cross section of temperature shown in Fig. 1. The very cold temperatures at polar latitudes are produced primarily by a balance between heat lost by radiation to space and latent heat released by the condensation of $\mathrm{CO}_{2}$, the Martian atmosphere's primary constituent. The lower midlatitudes are becoming warmer as Mars moves toward its vernal equinox, and the thermal contrast across high midlatitudes is particularly strong at this season. Mars rotates nearly as rapidly as does Earth, so that the winds at extratropical latitudes should be nearly in thermal wind balance. Figure 1 also shows the zonally averaged eastward winds that we have constructed from the zonally averaged temperature field derived by Conrath (1981). The gradient wind balance was used and the winds were assumed to vanish close to the Martian surface. Reasonably good fits to the gradient winds above 0.5 $\mathrm{mb}(\sim 20 \mathrm{~km})$ are given by (1) with $U_{0}$ in the range $200-240 \mathrm{~m} \mathrm{~s}^{-1}$ and with $B=20^{\circ}, \theta_{0}=60^{\circ}$. Somewhat better fits are given by slightly wider jets $\left(B=25^{\circ}\right.$, with $U_{0}=180-210 \mathrm{~m} \mathrm{~s}^{-1}$ ), but the averaging of retrieved temperatures within $10^{\circ}$ latitudinal bins may have artificially broadened the zonal jet. These values of $U_{0}$ combined with the Martian rotation rate and radius yield Rossby parameters in the range $0.35-0.5$; these values are significantly larger than those examined by Hartmann (1983). His largest $U_{0}$ value $\left(240 \mathrm{~m} \mathrm{~s}^{-1}\right)$ for Earth yields $R o=0.26$, which corresponds to $U_{0}=124$ $\mathrm{m} \mathrm{s}^{-1}$ for Mars (see Table 1). It was therefore necessary to solve (3) for the larger Ro values appropriate to Mars.
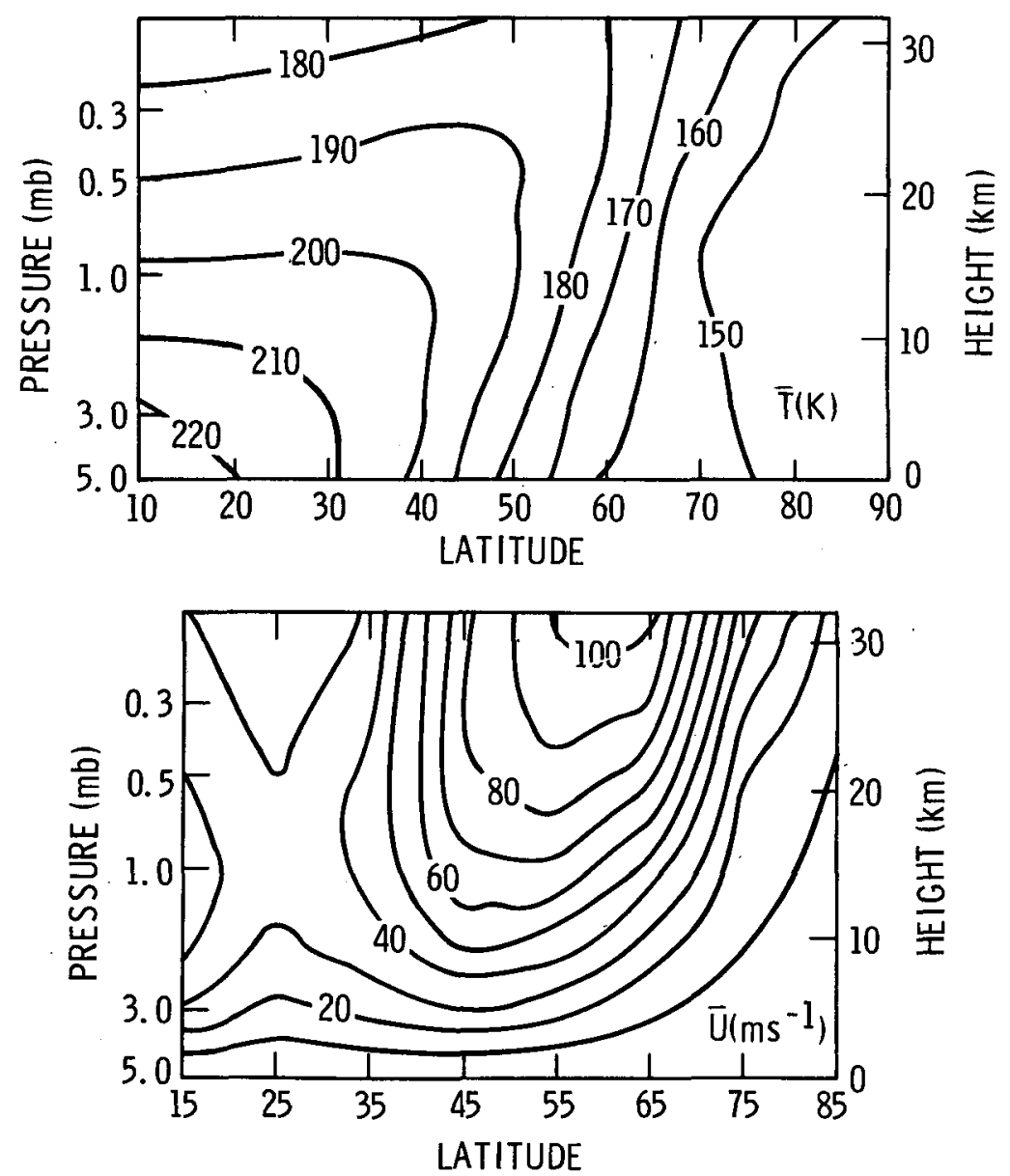

FIG. 1. (top) Observed mean meridional temperature $\bar{T}(\mathrm{~K})$ and (bottom) computed mean zonal gradient wind $\bar{u}\left(\mathrm{~m} \mathrm{~s}^{-1}\right)$ for northern winter on Mars. The temperature profiles used in constructing this cross section were taken from Conrath (1981), who analyzed the spectral radiances measured by the Mariner- 9 Infrared Interferometer Spectrometer (IRIS). The zonal gradient winds were calculated assuming that the wind vanished at the $5 \mathrm{mb}$ level, near the surface. 


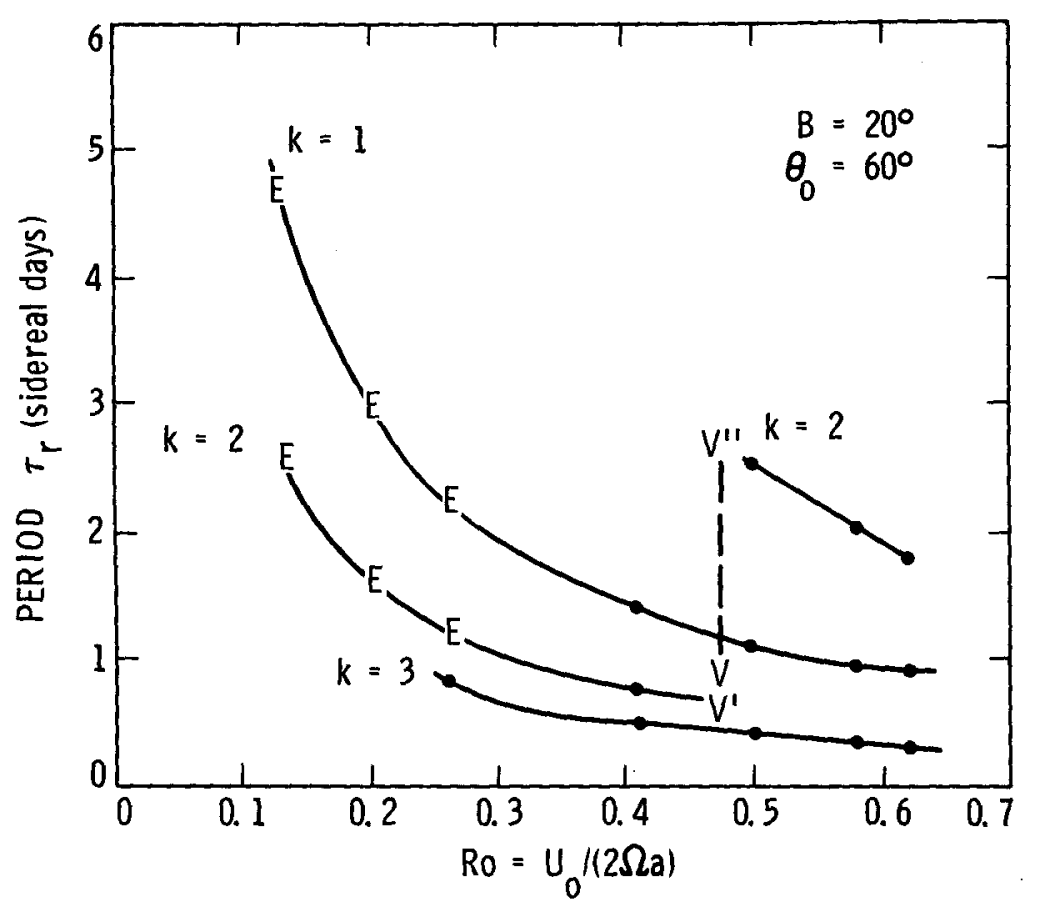

FIG. 2. Periods $\tau_{r}$ as a function of Ro for various zonal wavenumbers. The periods have been normalized by $\Delta$, the length of the sidereal day. The letter $E$ indicates values from Hartmann (1983). The letters $V^{\prime}, V^{\prime \prime}$ indicate Venus values derived for slightly different Ro values and the dashed line connecting them delineates a discontinuous transition for the $k=2$ curve. The letter $V$ denotes a Venus case derived from Elson's (1982) tabulated results.

\section{Barotropic instability: General results}

As noted earlier, the computed periods and $e$-folding times of the solution to the linearized, nondivergent barotropic vorticity equation depend only on $\mathrm{Ro}=U_{0} /$ $2 \Omega a$ for a given zonal wavenumber and for a fixed angular variation and position of the zonal jet, as long as the time scales are expressed in terms of the sidereal day $\Delta=2 \pi / \Omega$. Thus, we define the wave period $\tau_{r}$ $\equiv 2 \pi\left(k \sigma_{r} \Delta\right)^{-1}$ and the wave $e$-folding time $\tau_{i} \equiv\left(k \sigma_{i} \Delta\right)^{-1}$. This permits a unified presentation of Elson's (1982) results for Venus, Hartmann's (1983) results for Earth, and our results for Mars, with regard to the barotropic instability of high-latitude zonal jets. Figures 2 and 3 show the dependence on Ro of $\tau_{r}$ and $\tau_{i}$ for a given zonal wavenumber $k$. The points indicated by the letter $E$ are taken directly from Hartmann's (1983) tables and are representative of barotropic instability for the terrestrial polar night jet. As a test, the growth times and periods of the fastest growing waves for Ro $\sim 0.20$ and $0.26\left(U_{0}=180\right.$ and $\left.240 \mathrm{~m} \mathrm{~s}^{-1}\right)$ and with $k=1,2$ were exactly reproduced by our model. All cases shown in Figs. 2 and 3 used the zonal wind profile given by (1) with $\theta_{0}=60^{\circ}$ and $B=20^{\circ}$, except for the point for Venus indicated by the letter $V$. This was taken from Elson's (1982) Table 2 (the "low-latitude" model with $\theta_{0}=62^{\circ}$ and $k=2$ ) and normalized by a sidereal day
$\Delta_{V}=4 \Delta_{M}$ (i.e., a 4-day wind). The results labeled $V^{\prime}$ in Figs. 2 and 3 were computed using (1) with $U_{0}=100$ $\mathrm{m} \mathrm{s}^{-1}$, as suggested by Elson's representation of the mean zonal wind near the Venus cloud tops. The agreement between the points $V$ and $V^{\prime}$ is very good, considering the somewhat different latitudinal shapes assumed for the cyclostrophic Venus jet by Elson and by us. The value indicated by $V^{\prime \prime}$ in Fig. 2 is for a case having the same parameters as for case $V^{\prime}$, except that Ro is slightly larger. The discontinuous change in the wave period signifies a transition in which barotropically unstable modes on the equatorward flank of the jet grow more rapidly than those on the poleward flank, for zonal wavenumber $k=2$. This transition occurs at larger Ro for wider jets and, in fact, has moved between Ro $=0.62$ and 0.65 for $B=25^{\circ}$ (Fig. 4).

The sensitivity of the period and growth rate of the unstable waves to the width of the jet can be seen in Figs. 4 and 5. From Figs. 3 and 5, it is clear that the fastest growing wave is of wavenumber 1 or 2 , with the latter favored for narrower jets and for larger Ro. The $e$-folding times $\tau_{i}$ become quite short for large Ro, ranging from 1 to 3 days. The wave period $\tau_{r}$ decreases for larger zonal wavenumbers or as Ro increases, except for $k=2$ and $B<25^{\circ}$, where $\tau_{r}$ increases discontinuously by a factor of 4 or more. These longer period modes are members of the same family of midlatitude 


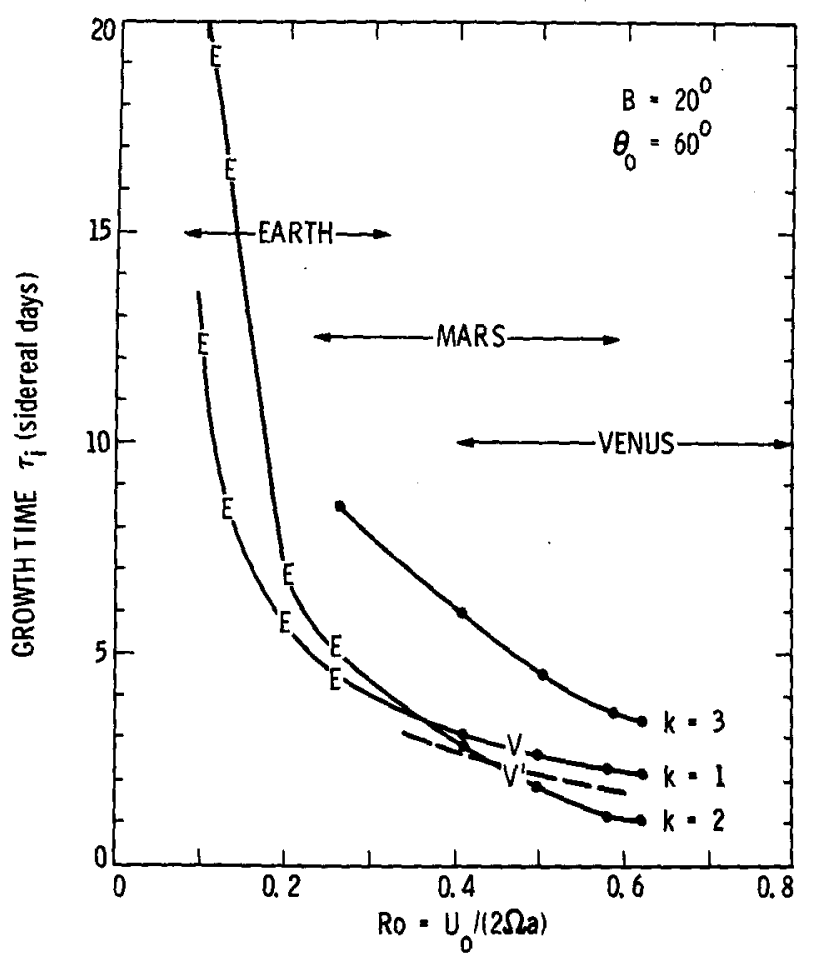

FIG. 3. As in Fig. 2, except for the $e$-folding times $\tau_{i}$, also normalized by $\Delta$. The dashed line through the value indicated by $V^{\prime}$ denotes a hypothetical solution in which the physical growth time $r_{i} \Delta_{V}$ would remain constant for Venus as $\Delta_{V}$, but not $U_{0}$, changes. The horizontal arrows depict typical ranges of Ro for Earth, Mars, and Venus.

modes studied by Hartmann (1983), except that for Ro $\geqslant 0.45$ and for jets which are not too broad, these midlatitude modes become the fastest growing baro- tropic instabilities. Thus, for $B=20^{\circ}$ and $\theta=60^{\circ}$, the fastest growing mode shifts as Ro increases from a polar $k=1$ wave to a shorter period $k=2$ polar mode and then to a longer period, $k=2$ midlatitude mode. For a sufficiently broad jet $\left(B=25^{\circ}\right)$, the polar $k=1$ and $k=2$ modes may continue to dominate as Ro increases (Figs. 4 and 5). The position of the jet $\left(\theta_{0}\right)$ was also varied, and the results are listed in Table 3 . The sensitivity of $\tau_{i}$ to $\theta_{0}$ is comparable to that for $B$, the jet width, while $\tau_{r}$ is more sensitive to $\theta_{0}$. Again, this is because of a shift from a poleward to an equatorward mode as the jet maximum is moved to a lower latitude. In this sense, a shift to lower latitude has the same effect as narrowing the jet. The contrast between the poleward modes and the longer period, midlatitude modes is clearly evident in Figs. 6,7 and 8, where the perturbation streamfunction amplitude structure and the wave-induced acceleration $A_{u}$, together with the basic state $\bar{u}$ and $\alpha$, are shown, all scaled to arbitrary amplitudes. For both the poleward and equatorward modes, the wave-induced zonal forcing per unit mass is such that the zonal jet is being deaccelerated near its maximum and accelerated on the poleward and equatorward flanks, respectively.

\section{Barotropic instability: Specific results}

\section{a. Venus}

In Fig. 6, the perturbation streamfunction $\psi$ has its maximum amplitude near $70^{\circ}$, poleward of the jet maximum. This mode is similar to the fastest growing wave computed by Elson (1982) for Venus and to the

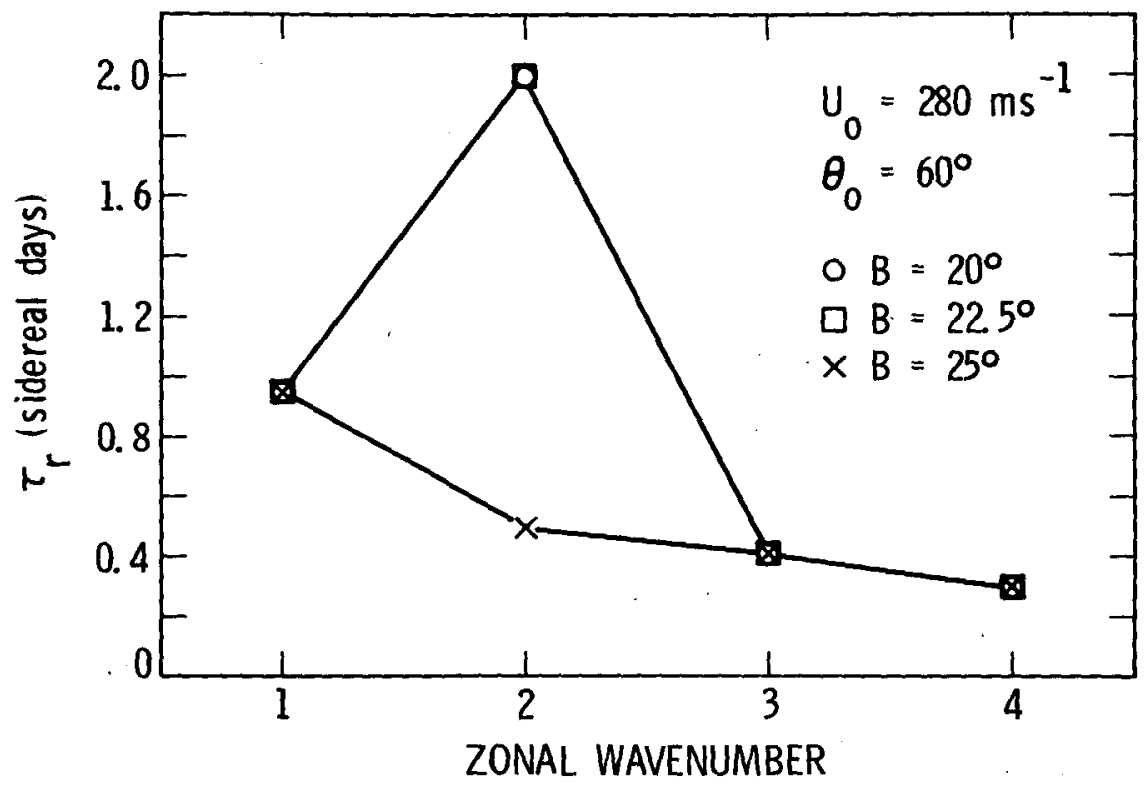

FIG. 4. Normalized periods $\tau_{r}$ for Mars as a function of zonal wavenumber, showing the effects of varying the width of the zonal jet. 


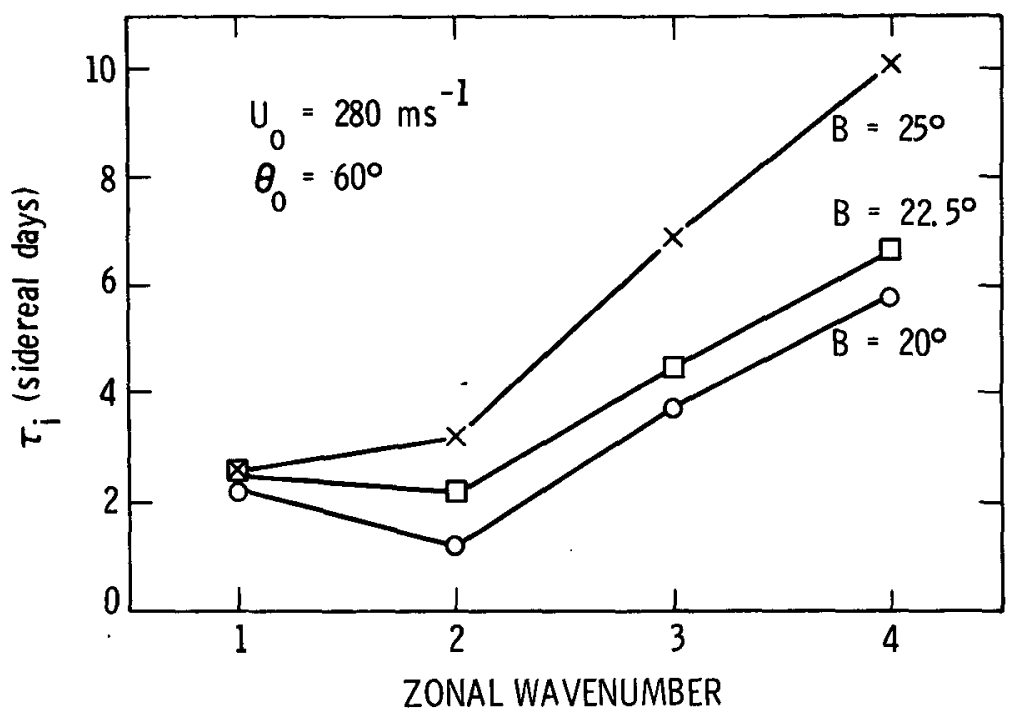

FIG. 5. Normalized growth times $\tau_{i}$ for the same cases as in Fig. 4.

poleward modes found by Hartmann (compare Fig. 6, for instance, with his Fig. 5). Venus appears perilously close to the transition between poleward and equatorward modes (Figs. 6 and 7). Since the jet is unlikely to remain constant in time or with latitude, the "dipole" thermal feature on Venus may change substantially with time. For situations characterized by higher Ro, the barotropic "dipole" should move equatorward to the vicinity of the zonal jet and have a much longer period ( $\sim 10$ Earth days). For sufficiently lower Ro, the dipole should transform into a $k=1$ pattern, still at high latitude, but again with a long period $(\sim 6$ Earth days).

The normalization of the growth times and periods by $\Delta$ may be misleading for slowly rotating planets like Venus where $\Delta_{V}$ is the solid-body component of an atmospheric rotation which varies with height. If the characteristic angular velocity of the jet decreases as rapidly with height as the Venus atmosphere's solidbody rotation, then the middle atmosphere will have constant Ro, $\tau_{i}$, and $\tau_{r}$, while the actual growth times $\left(\tau_{i} \Delta\right)$ and periods $\left(\tau_{r} \Delta\right)$ will increase with $\Delta$ and thus with height. In this case, the fastest growing barotropic waves would be near the cloud top region where the thermal polar "dipole" was observed (Taylor et al.,

TABLE 3. The $e$-folding times $\left(\tau_{i}\right)$ and periods $\left(\tau_{r}\right)$, given in sidereal days, for the fastest growing barotropic wave for various positions $\left(\theta_{0}\right)$ of the jet maximum and for Ro $=0.58, B=22.5^{\circ}, k=2$. The latitude $\theta_{*}$ of the maximum in streamfunction amplitude is also given.

\begin{tabular}{llll}
\hline \hline$\theta_{0}$ & $\tau_{i}$ & $\tau_{r}$ & $\theta_{*}$ \\
\hline $45^{\circ}$ & 1.1 & 1.3 & $42^{\circ}$ \\
$60^{\circ}$ & 2.2 & 2.0 & $54^{\circ}$ \\
$70^{\circ}$ & 6.4 & 0.5 & $78^{\circ}$ \\
\hline
\end{tabular}

1980). The dashed curve through the point labeled $V^{\prime}$ in Fig. 3 shows a hypothetical solution for $\tau_{i}$ in which $\tau_{i} \Delta$ remains constant for $k=2$ even as the atmospheric rotational period $\Delta$, but not the relative angular velocity of the zonal jet, changes. Since the slope of the $k=2$ curve is steeper than that of this hypothetical solution near the current Venus solution, the dimensional, as well as the nondimensional, growth time will decrease with height, for this case of nearly constant $U_{0}$. This suggests that barotropically unstable modes could grow faster near $75 \mathrm{~km}$ than at the visible cloud tops near $65 \mathrm{~km}$, but ignores possible effects of the vertical shear itself. If the barotropic zonal jet remains relatively narrow, the mode at $75 \mathrm{~km}$ should have its maximum amplitude on the equatorward side of the jet and its actual period in the range 8-10 Earth days. The thermal structure of an unstable wave having these characteristics was not detected in the 72-day Pioneer Venus OIR data record (Apt and Leung, 1982). Above $75 \mathrm{~km}$ on Venus, the rotational period $\Delta_{V}$ may become very large (Elson, 1978; Newman et al., 1984). Eventually, the jet, too, will decay with height and the actual growth time will begin to increase. Thus, barotropically unstable waves seem less likely to be present in the upper regions of the Venus middle atmosphere.

\section{b. Mars}

The gradient wind shown in Fig. 1 has considerable vertical structure. In order to check whether or not the atmosphere away from the surface was itself baroclinically unstable, the contribution of the vertical shear and curvature terms to changing the sign of the meridional gradient of quasi-geostrophic potential vorticity was computed for the fields shown in Fig. 1. Except near the surface, this contribution was small compared 


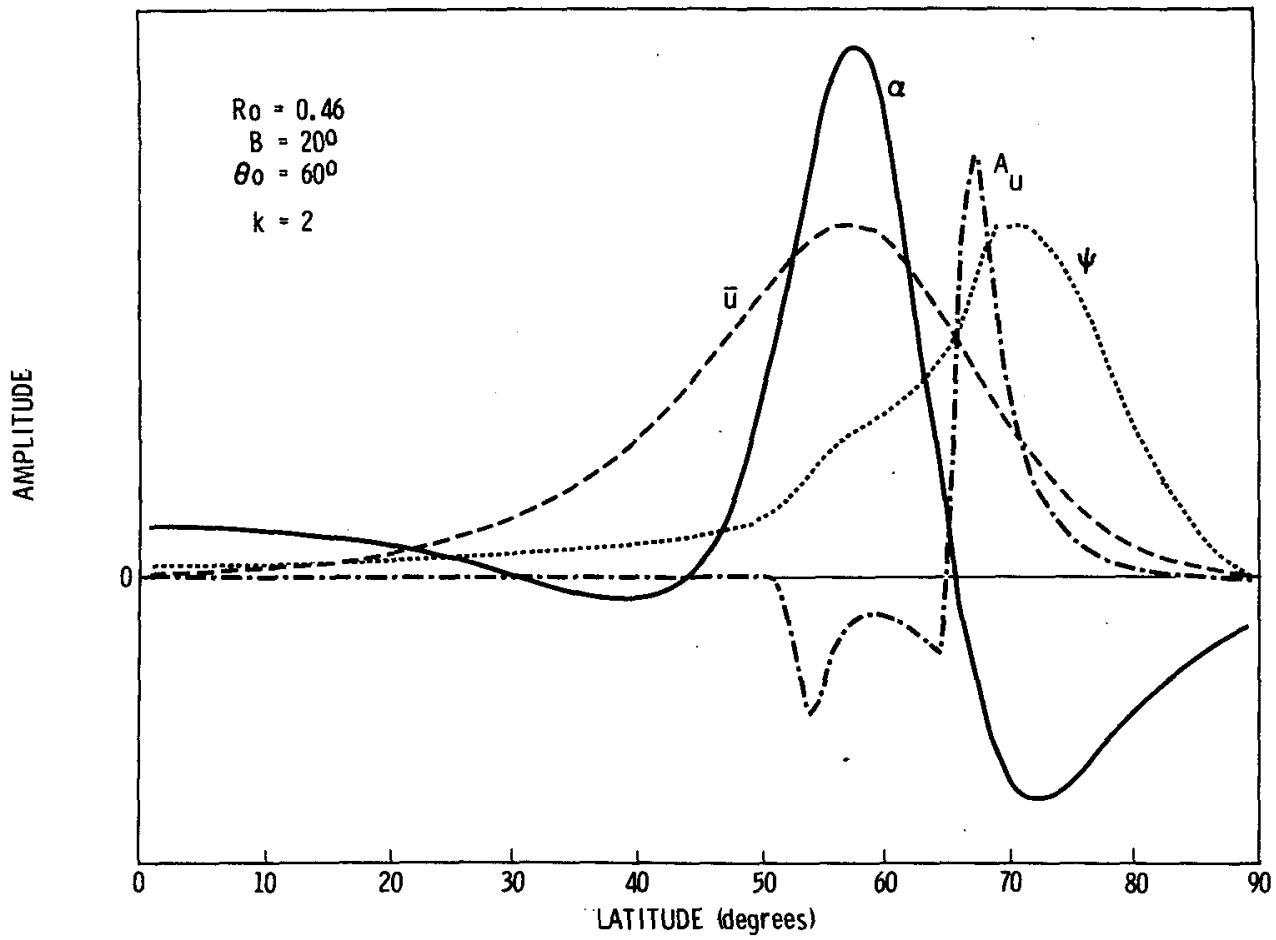

Fig. 6. Structure of the most unstable barotropic mode for $\bar{u}$ given by Eq. (1) with $\theta_{0}=60^{\circ}, B=20^{\circ}$ and Ro $=0.46$. For this mode, $k=2, \tau_{r}=0.7$ days and $\tau_{i}=2.5$ days. The zonal mean wind $\vec{u}$, basic state vorticity gradient $\alpha$, perturbation streamfunction amplitude $|\psi|$ and wave-induced acceleration $A_{u}$ are given in arbitrary units.

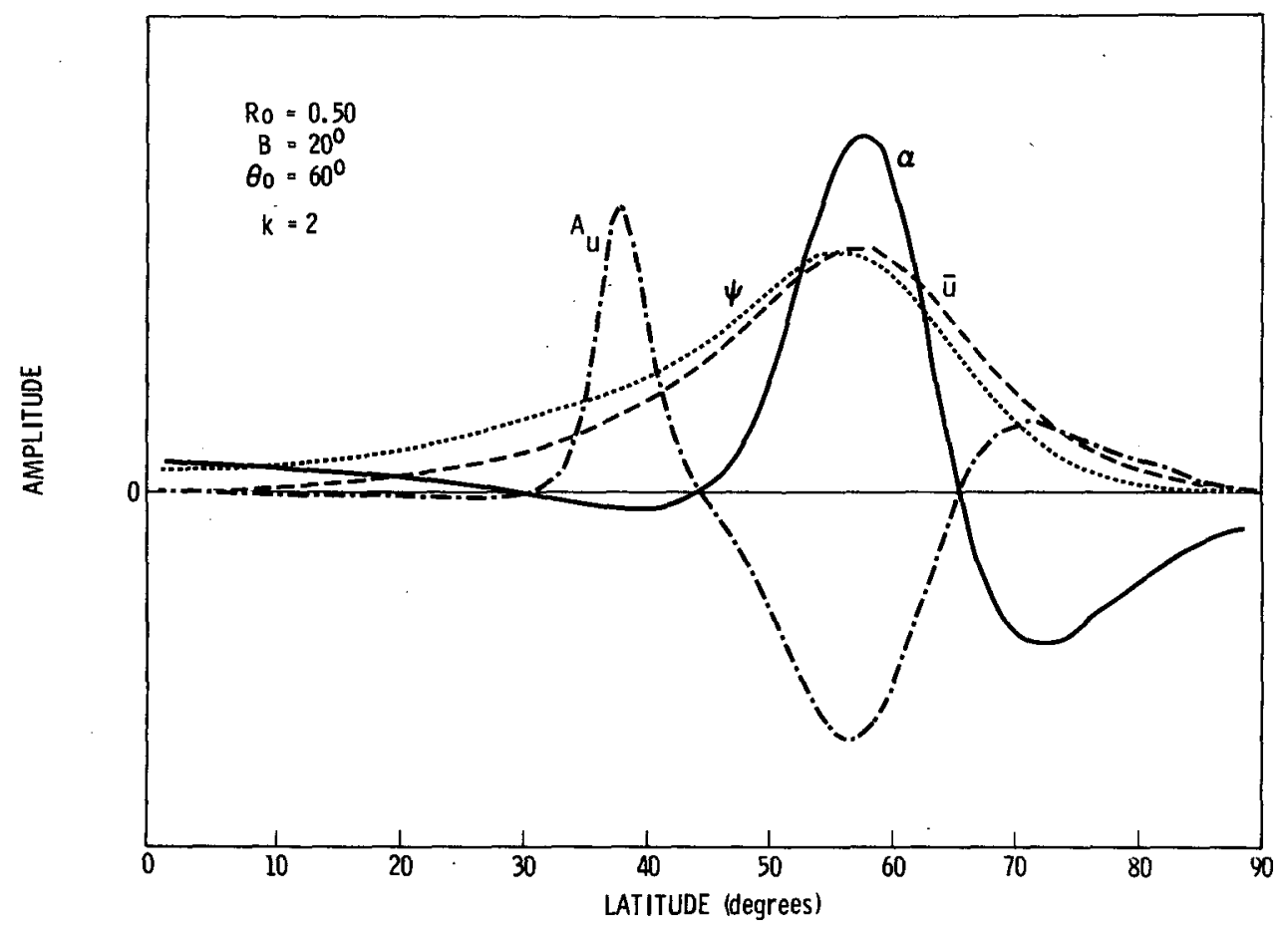

FIG. 7. As in Fig. 6, except Ro $=0.50, \tau_{r}=2.5$ days and $\tau_{i}=2.0$ days. Note the equatorward shift of $\psi$ and of the wave-induced acceleration $A_{u}$. 


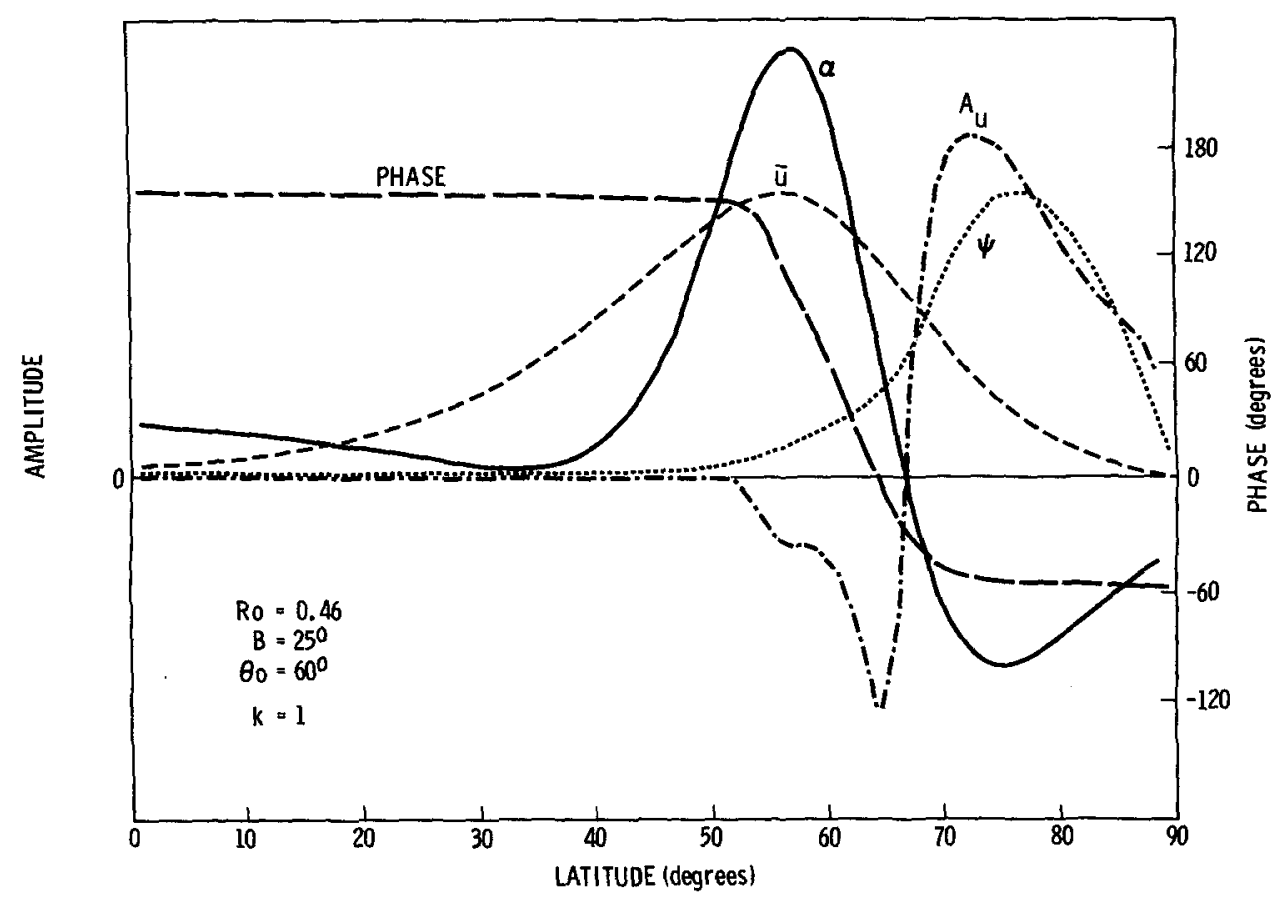

FiG. 8. As in Fig. 6, except for $B=25^{\circ}$. For this mode $k=1, \tau_{r}=1.2$ days and $\tau_{i}=3.2$ days. The phase of the streamfunction is also shown.

to that of the meridional gradient of absolute vorticity. Of course, the presence of a latitudinal gradient of potential temperature at the surface can still produce baroclinically unstable waves (Charney and Stern, 1962; Barnes, 1984). Barnes (1980, 1981) found strong evidence in the Viking Lander-2 surface wind, temperature, and pressure data, for eastward-traveling, quasi-geostrophic waves of zonal wavenumbers between 2 and 4 at $48^{\circ} \mathrm{N}$. The corresponding periods were between 8 and 2 Martian days (sols). These observed values are inconsistent with the barotropic wave parameters computed here, but that does not preclude the presence of waves at higher altitudes which are largely barotropic in nature. Pfister (1979) showed that the vertical shear of $\bar{u}$ will tend to confine barotropic modes near the levels of strongest horizontal shear. For Mars and the winds shown in Fig. 1, this means that the barotropically unstable modes should have little amplitude at the surface.

Conrath's (1981) wave analysis of the Mariner-9 temperature data implied long periods of 10 days or more for low wavenumbers, consistent with the analyses by Barnes $(1980,1984)$ of the Viking Lander meteorological data. However, the Mariner-9 data were restricted by the spacecraft orbit and limitations in data transmission to sampling in time just once per day. As pointed out clearly by Conrath, this infrequent sampling could have led to serious aliasing of the data, so that there were many well-defined but disjoint fre- quencies which were consistent with the observations for a given zonal wavenumber.

For wavenumbers 1 and 2, which are barotropically the fastest growing waves for the Mars midlatitude jet, the first-order alias of Conrath's analysis yields eastward traveling waves with periods of 1.24 or 1.105 days for $k=1$, and 1.20 or 1.07 days for $k=2$. In Fig. 2, these periods correspond to an Ro of 0.46 or 0.5 for $k=1$ and to 0.26 or 0.29 for $k=2$. For the zonal jet shown in Fig. 1, these two Ro ranges occur above the 0.3 and near the $1.0 \mathrm{mb}$ levels, respectively. Conrath (1981) found that the largest temperature wave observed by Mariner-9 had its maximum amplitude in the region between 1.5 and $0.6 \mathrm{mb}$, where the $k=2$ mode has the correct period. However, in this Ro range $(0.26-$ 0.31 ), the $k=1$ mode should grow somewhat faster than the $k=2$ wave (see Fig. 3). In fact, the structure of the primary temperature wave identified by Conrath is characteristic below $0.5 \mathrm{mb}$ of a vertically propagating wave, perhaps generated by baroclinic instability at the surface. Both Conrath's calculation of the index of refraction for a vertically propagating wave and the linear baroclinic instability study by Barnes (1984) indicate that such a wave would have little temperature amplitude at $0.5 \mathrm{mb}$ and, indeed the observed phase of the wave ceases to tilt westward with height near this level. However, the observations suggest that there is still sizeable amplitude above this level where the fastest growing barotropically unstable wave is a $k=1$ 
mode with the proper period. Such a mode is shown in Fig. 8.

Hartmann's (1983) results indicate that the latitude of maximum temperature amplitude for the quasigeostrophic barotropic modes for Earth will be close to that of the maximum streamfunction amplitude for the nondivergent modes. If so, the poleward mode shown in Fig. 8 could not account for the temperature variation found by Conrath for the $40^{\circ}-70^{\circ}$ latitudinal zone. Midlatitude (i.e., $k=2$ ) modes have their maximum at the right latitudes (Fig. 7), and would be the fastest growing barotropic modes for a narrower zonal jet (Fig. 3). However, their periods are too long. Faster growing midlatitude barotropic modes may also occur above the $0.1 \mathrm{mb}$ level if the wind continues to increase with height. The thermal signature of these modes may depend critically on the radiative damping time, which should decrease rapidly with height in this region. Our results do suggest that the character of the fastest growing barotropic mode in the middle Martian atmosphere may change significantly (e.g., the maximum amplitude may shift from the poleward to the equatorward flank) as the width of the zonal jet changes seasonally or from year to year. During a great dust storm on Mars, for instance, the zonal jet near the northern polar night may be narrowed and shifted poleward. As noted in section 4, these are competing effects. The Mariner-9 data used to construct Fig. 1 were taken, in fact, during the final stages of one of the most global of these dust storms.

\section{Conclusion}

Barotropically unstable, relatively broad, high-latitude zonal jets appear to be present on all three of the inner planets having atmospheres of any consequence: Earth, Mars, and Venus. The unstable modes derived here for the Martian polar night jet can be easily related parametrically to the Venus modes found by Elson (1982) and to the terrestrial modes computed by Hartmann (1983), since all solutions to the linearized, nondivergent barotropic vorticity equation depend primarily on the Rossby number of the zonal jet, when the width of the jet is itself planetary-scale. Both Mars, with its fast circumpolar jet and small planetary radius, and Venus, with its slower rotation rate, have characteristically larger Ro than does Earth, but the families of barotropic modes are extensions of those studied by Hartmann (1983) for the terrestrial stratosphere. As Ro increases into the range appropriate for Mars and Venus, the fastest growing barotropic mode shifts first from a polar $k=1$ mode to a shorter period, but still polar, $k=2$ mode and then possibly to a midlatitude, longer period $k=2$ mode. Thus, unstable modes having zonal wavenumber $k=2$ and periods of $1-3$ sidereal days are more likely to be the dominant barotropic modes on Mars and Venus, than on Earth, where modes having $k=1$ and periods of 2-4 days seem more likely to appear. Furthermore, for the hyperbolic jet profiles examined here, the existence for Mars and Venus of two families of unstable modes having comparable growth rates but different periods and generated on opposite sides of the zonal jet implies that there may be greater variability in planetary-scale wave characteristics near the midlatitude zonal jets on these two planets than on Earth.

Whether or not these barotropic modes or their quasi-divergent counterparts can directly account for the temperature waves seen in the satellite radiance data for Venus, Earth, and Mars remains to be seen. Hartmann has suggested that the primary effect of barotropic instability may be to interact strongly with and to locally enhance planetary waves propagating from below. This may be particularly true for Mars where baroclinic instability at and near the surface is known to generate waves of planetary-scale at high latitudes (Barnes, 1984). The combination of the zonal wind and the horizontal wind associated with barotropically unstable waves may also produce for Mars a longitudinally varying waveguide which modulates the ability of other planetary-scale waves-however they are produced-to propagate to the levels observed. The case for Venus is, as usual, more enigmatic. In all cases, the final determination must include the effects of divergence and of the vertical, as well as meridional, variations of the high-latitude zonal jets that are present in each of the three planetary atmospheres.

Acknowledgments. The authors wish to acknowledge an anonymous reviewer who pointed out the importance of the ageostrophic correction to the zonal-mean zonal wind computed for Mars. This research was sponsored by NASA's Planetary Atmospheres Program and was performed in part at the Jet Propulsion Laboratory, California Institute of Technology, under contract with the National Aeronautics and Space Administration.

\section{REFERENCES}

Apt, J., and J. Leung, 1982: Thermal periodicities in the Venus atmosphere. Icarus, 49, 427-437.

Barnes, J. R., 1980: Time spectral analysis of midlatitude disturbances in the Martian atmosphere. J. Atmos. Sci., 37, 2002-2015.

- 1981: Midlatitude disturbances in the Martian atmosphere: A second Mars year. J. Atmos. Sci., 38, 225-234.

- 1984: Linear baroclinic instability in the Martian atmosphere. J. Atmos. Sci., 41, 1536-1550.

Charney, J. G., and M. Stern, 1962: On the stability of internal baroclinic jets in a rotating atmosphere. J. Atmos. Sci., 19, 159-172.

Conrath, B. J., 1981: Planetary-scale wave structure in the Martian atmosphere. Icarus, 48, 246-255.

Elson, L. S., 1978: Barotropic instability in the upper atmosphere of Venus. Geophys. Res. Lett., 5, 603-605.

- 1982: Wave instability in the polar region of Venus. J. Atmos. Sci., 39, 2356-2362. 
Hartmann, D. L., 1983: Barotropic instability of the polar night jet stream. J. Atmos. Sci., 40, 817-835.

Kuo, H.-L., 1949: Dynamic instability of two-dimensional nondivergent flow in the barotropic atmosphere. J. Meteor., 6, 105122

Martin, T. Z., and H. H. Kieffer, 1979: Thermal infrared properties of the Martian atmosphere. 2. The $15 \mu \mathrm{m}$ band measurements. J. Geophys. Res., 84, 2843-2852.

Newman, M., G. Schubert, A. J. Kliore and I. R. Patel, 1984: Zonal winds in the middle atmosphere of Venus from Pioneer Venus radio occultation data. J. Atmos. Sci., 41, 1901-1913.

Pfister, L., 1979: A theoretical study of three-dimensional barotropic instability with applications to the upper stratosphere. J. Atmos. Sci., 36, 908-920.

Taylor, F. W., J. T. Schofield and P. J. Valdes, 1985: Temperature structure and dynamics of the middle atmosphere of Venus. Adv. Space Res., 5, 5-23.

—, R. Beer, M. T. Chahine, D. J. Diner, L. S. Elson, R. D. Haskins, D. J. McCleese, J. V. Martonchik, P. E. Reichley, S. P. Bradley, J. Delderfield, J. T. Schofield, C. B. Farmer, L. Froidevaux, J. Leung, M. T. Coffey and J. C. Gille, 1980: Structure and meteorology of the middle atmosphere of Venus: Infrared remote sensing from the Pioneer Orbiter. J. Geophys. Res., 85, 79638006.

Venne, D. E., and J. L. Stanford, 1979: Observation of a 4-day temperature wave in the polar winter stratosphere. J. Atmos. Sci., 36, 2016-2019.

- , and — 1982: An observational study of high-latitude stratospheric planetary waves in winter. J. Atmos. Sci., 39, 10261034. 